\title{
(2) OPEN ACCESS \\ Quality of investigations into unexpected deaths of infants and young children in England after implementation of national child death review procedures in 2008: a retrospective assessment
}

\author{
Peter Fleming (ㄷ, , Anna Pease, ${ }^{1}$ Jenny Ingram 다, ${ }^{1}$ Peter Sidebotham, ${ }^{2}$ \\ Marta C Cohen (1) , ${ }^{3}$ Robert C Coombs, ${ }^{4}$ Andrew K Ewer (1) , ${ }^{5}$ Martin Ward Platt (1) , ${ }^{6}$ \\ John Fox, ${ }^{7}$ David Marshall, ${ }^{8}$ Anne Lewis, ${ }^{9}$ Carol Evason-Coombe, ${ }^{10}$ Peter Blair ${ }^{1}$
}

\begin{abstract}
- Additional material is published online only. To view please visit the journal online (http://dx.doi.org/10.1136/ archdischild-2019-317420).

For numbered affiliations see end of article.
\end{abstract}

\section{Correspondence to} Dr Peter Fleming, Centre for Academic Child Health, University of Bristol, Bristol BS2 8EG, UK:

Peter.Fleming@bristol.ac.uk

Received 15 April 2019 Revised 26 August 2019 Accepted 2 September 2019 Published Online First 27 September 2019

\section{Check for updates}

(C) Author(s) (or their employer(s)) 2020. Re-use permitted under CC BY-NC. No commercial re-use. See rights and permissions. Published by BMJ.

To cite: Fleming $P$, Pease $A$, Ingram J, et al. Arch Dis Child 2020;105:270-275

\begin{abstract}
Objectives In 2008, new statutory national procedures for responding to unexpected child deaths were introduced throughout England. There has, to date, been no national audit of these procedures.

Study design Families bereaved by the unexpected death of a child under 4 years of age since 2008 were invited to participate. Factors contributing to the death and investigations after the death were explored. Telephone interviews were conducted, and coroners' documents were obtained. The nature and quality of investigations was compared with the required procedures; information on each case was reviewed by a multiagency panel; and the death was categorised using the Avon clinicopathological classification.

Results Data were obtained from 91 bereaved families (64 infant deaths and 27 children aged $1-3$ years); 85 remained unexplained after postmortem examination. Documentation of multiagency assessments was poorly recorded. Most (88\%) families received a home visit from the police, but few $(37 \%)$ received joint visits by police and healthcare professionals. Postmortem examinations closely followed national guidance; $94 \%$ involved paediatric pathologists; $61 \%$ of families had a final meeting with a paediatrician to explain the investigation outcome. There was no improvement in frequency of home visits by health professionals or final meetings with paediatricians between 2008-2013 and 2014-2017 and no improvement in parental satisfaction with the process. Conclusions Statutory procedures need to be followed more closely. The implementation of a national child mortality database from 2019 will allow continuing audit of the quality of investigations after unexpected child deaths. An important area amenable to improvement is increased involvement by paediatricians.
\end{abstract}

\section{INTRODUCTION}

In 2003, after the acquittal of several mothers convicted of murdering their babies, a multiprofessional working group devised a multiagency approach to the investigation of unexpected infant deaths, including a standard postmortem protocol. $^{12}$ This was incorporated into the statutory guidance to the Children Act $2004 .^{3}$

In 2008, this statutory programme was implemented in England aiming to ensure appropriate

\section{What is already known on this topic?}

- Statutory guidance for the investigation of sudden unexpected deaths in children was introduced in the England in 2008.

- The degree to which this guidance is followed has not previously been ascertained.

\section{What this study adds?}

- The guidance for investigating sudden unexpected deaths is still only partially implemented by professionals.

- Classification of deaths is inconsistent.

- There is wide variation in the quality of care and support provided to bereaved families that cannot yet be said to be a truly family-centred process.

investigation and support of families to reduce wrongful accusations while ensuring the recognition of genuine maltreatment. The recommendations emphasised the importance of multiagency collaboration, data sharing and involvement with a final case review meeting involving all agencies to assess the likely cause of and any contributory factors to the death.

A national training programme for professionals was implemented, together with a joint visit to the scene of the death by the police and a paediatrician or trained nurse.

We report data collected on the nature and quality of investigations after unexpected infant and child deaths in England since the implementation of these programmes.

\section{METHODS}

\section{Recruitment of families}

The Lullaby Trust, a charity providing support to families bereaved by unexpected infant death, funds research into such deaths in England.

From July 2016 to October 2017, families were invited to contact the Lullaby Trust if their infant or child aged under 4 years had died unexpectedly since 2008. Families interested in taking part were 


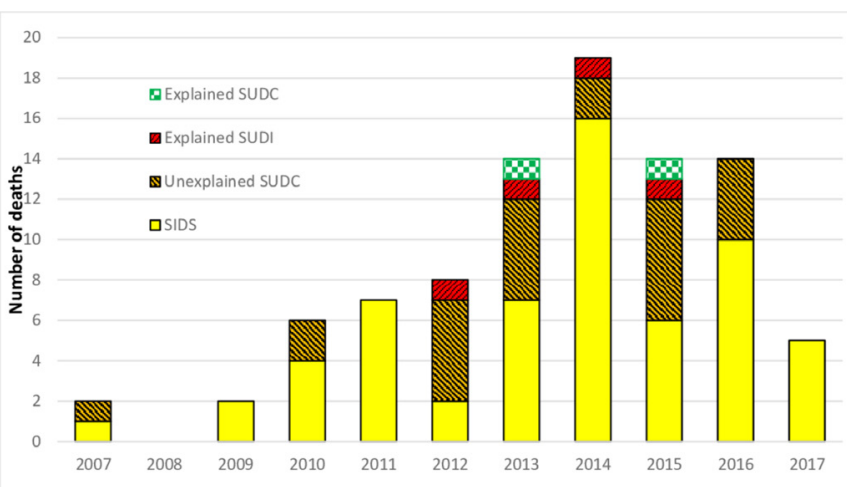

Figure 1 Year of sudden unexpected death (91 deaths). SIDS, sudden Infant death syndrome; SUDC, sudden unexplained death in childhood.

contacted by their preferred modality (email, telephone and text) to obtain written consent to participate in the study, including consent to request information from the coroner. A semistructured telephone interview was conducted to collect questionnaire data with an opportunity for parents to give their account in their own words (data capture form is shown in online supplementary appendix I).

\section{Obtaining the documentation}

Deliberations by Child Death Overview Panels are legally privileged (not disclosable), but the results of the local child death review process should be fully disclosed to the inquest. ${ }^{3-5} \mathrm{We}$ therefore collected data from coroners as these data are potentially in the public domain (with appropriate consent).

We contacted coroners' offices seeking copies of all inquest documentation, including the police report, paediatrician's report, postmortem report and the report of the final child death review meeting. All coroners contacted (with evidence of parental informed consent) gave us all available documentation.

\section{Multiprofessional case reviews}

The multiprofessional teleconference case review meetings included experienced professionals in the field (paediatrician, paediatric pathologist, health visitor, police officer and social worker). Four to six cases were discussed and categorised at each meeting (90-120 min). Panel members were sent a detailed, anonymised copy and structured summary of all available data on each death and were asked to prepare a provisional Avon classification of any relevant possibly contributory factors ${ }^{3}$ before the meeting.

The chair had the original (non-anonymised) documents available to clarify any questions arising during the discussion, but great care was taken to protect anonymity in the discussions. Each case was categorised by the panel according to the Avon clinicopathological categorisation system, ${ }^{3}$ and any differences in opinion regarding the classification of death were decided by majority opinion.

\section{Data handling}

The information documented in each case was compared with the recommended minimum investigations, care of the family and clinical and pathology protocol defined in the multiagency guidelines ${ }^{4}$ for management of unexpected child deaths. Findings in the period 2008-2013 were compared with 2014-2017 to identify any changes over the 10 -year period. Descriptive
Table 1 Avon classification of sudden unexpected deaths

\begin{tabular}{|c|c|c|c|}
\hline \multicolumn{4}{|c|}{ Sudden unexpected death in infancy (SUDI) $(n=64)$} \\
\hline 0 & Information not collected & $0 / 64$ & $0 \%$ \\
\hline IA & $\begin{array}{l}\text { No potentially significant factors or contributory } \\
\text { factors found (SIDS) }\end{array}$ & $0 / 64$ & $0 \%$ \\
\hline IB & $\begin{array}{l}\text { Factors present but not likely to have contributed } \\
\text { to the death (SIDS) }\end{array}$ & $10 / 64$ & $15.6 \%$ \\
\hline$\| \mathrm{A}$ & $\begin{array}{l}\text { Factors present and may have contributed to the } \\
\text { death (SIDS) }\end{array}$ & 29/64 & $45.3 \%$ \\
\hline IIB & $\begin{array}{l}\text { Factors present and probably contributed to the } \\
\text { death (SIDS) }\end{array}$ & $21 / 64$ & $32.8 \%$ \\
\hline III & Fully explained death & $4 / 64$ & $6.3 \%$ \\
\hline \multicolumn{4}{|c|}{ Sudden unexpected death in childhood $(n=27)$} \\
\hline 0 & Information not collected & $0 / 27$ & $0 \%$ \\
\hline IA & $\begin{array}{l}\text { No potentially significant contributory factors } \\
\text { found (SUDC) }\end{array}$ & $2 / 27$ & $7.4 \%$ \\
\hline IB & $\begin{array}{l}\text { Factors present but not likely to have contributed } \\
\text { to the death (SUDC) }\end{array}$ & $0 / 27$ & $0 \%$ \\
\hline$\| \mathrm{A}$ & $\begin{array}{l}\text { Factors present and may have contributed to the } \\
\text { death (SUDC) }\end{array}$ & $15 / 27$ & $55.6 \%$ \\
\hline IIIB & $\begin{array}{l}\text { Factors present and probably contributed to the } \\
\text { death (SUDC) }\end{array}$ & $8 / 27$ & $29.6 \%$ \\
\hline III & Fully explained death & $2 / 27$ & $7.4 \%$ \\
\hline
\end{tabular}

SIDS, sudden infant death syndromeSUDC, sudden unexplained death in childhood.

statistics are presented with numbers and percentages, medians and IQR where appropriate.

Parents were asked (using a 5-point Likert scale) to give their satisfaction with the overall investigation and the care they had received (see Appendix 1).

\section{RESULTS}

\section{Ascertainment}

Of the 102 bereaved families who contacted the Lullaby Trust, 92 completed the telephone interview and 1 family later withdrew consent. The deaths included 64 infants aged under 12 months (median age $=82$ days (IQR: 46-147 days)) and 27 aged 12 -41 months (median age $=610$ days (IQR: 477-676 days)).

Two families volunteered for the study whose child died in 2007 (one infant and one older child). Both were included in the study as they lived in areas in which the full recommended protocol had commenced before 2007.

Enough information from the parental interview, postmortem examination, clinical history and the death scene was available to the multidisciplinary panels for them to attribute an Avon classification $^{2}$ for all 91 deaths (table 1). For 83/91 (91\%) of the deaths, possible contributory factors were identified (eg, mild infections and potentially hazardous sleep environment) that did not fully explain the deaths; thus, meeting the definition of sudden infant death syndrome (SIDS) ${ }^{6}$ or sudden unexplained death in childhood (SUDC). ${ }^{7}$ Only six $(6.6 \%)$ were deemed to be fully explained (from previously unrecognised cardiac abnormalities or overwhelming infections).

There were only two classification discrepancies between panel members; all others (97.8\%) were unanimous.

The dataset includes data on 60 unexplained deaths of infants under 1 year of age (ie, SIDS) and 25 unexplained deaths of children aged 1-3 years (ie, SUDC); the latter group includes two children who died while overseas after 2014 and were excluded from the review of procedures after death, leaving 89 deaths included. 
Table 2 Documentation of visit to home or scene of death/collapse for 89 unexpected deaths

\begin{tabular}{ll}
\hline Agency visiting & $\begin{array}{l}\text { Cases in which visit was documented } \\
\mathbf{n}(\% \text { of total) }\end{array}$ \\
\hline Any home visit & $78(88)$ \\
\hline Police alone & $46(52)$ \\
\hline Health professional alone & 1 \\
\hline Social care alone & 0 \\
\hline Police+health professional & $32(36)$ \\
\hline Police+social care & 1 \\
\hline Police+health+social care & 1 \\
\hline Parents present for the home visit & 71 (91\% of home visits) \\
\hline Parents not present for the visit & 7 (9\% of home visits) \\
\hline
\end{tabular}

Figure 1 shows the years in which the deaths occurred. With approximately 2000 unexplained (SIDS) deaths of infants $\left(<1\right.$ year) in England in those years, ${ }^{8}$ our sample was around 3\% of the total SIDS population.

From 2010 to 2016, The Office for National Statistics (ONS) estimated there were 91 SUDC between the ages of 12 and 24 months in England and Wales. ${ }^{8}$ Our sample includes 18 SUDC deaths during this period and age range representing approximately 20\% of SUDC deaths in England and Wales.

The 39 deaths from 2007 to 2013 and 50 deaths from 2014 to 2017 have been used to identify any changes in care or investigations over this 10 -year period.

\section{Quality of care at the emergency department (ED)}

All 89 infants and children were taken to an ED with facilities for children. A paediatrician took a history in ED from $69 \%$ (61/89) of the parents and 78\% (69/89) families reported having seen a paediatrician at some stage. Contact between police and clinical staff in the ED was poorly documented. Most families reported seeing the police in the $\mathrm{ED}$, but few could remember whether the police and medical staff took a joint history, and coroner's documents commonly did not clarify this.

\section{Quality of care at scene of death}

Table 2 shows home visits to families and which agencies were involved, but only $38 \%$ families $(34 / 89)$ had a home visit by a healthcare professional. Before 2014, 44\% (17/39) of families had a home visit by a health professional compared with $34 \%$ $(17 / 50)$ after 2014. Many families spontaneously reported that they particularly valued this healthcare professional home visit.

Crucial information on the scene and circumstances of the death were commonly poorly documented, for example, exactly where baby had been sleeping or who was in what position in the bed for a death during cosleeping. Baby's sleeping position (prone/supine/side) was not always documented. Arrangements of bedding were commonly poorly described, and bedding had sometimes been removed by police before the home visit.

Very few follow-up multiagency discussion meetings after the initial meeting in the ED were documented (only two cases). Five sets of parents reported having been interviewed separately and had not been allowed to be with each other in the immediate aftermath of their baby's death.

\section{Quality of care relating to the pathology and interprofessional communication}

Paediatricians' reports to the coroner and/or the pathologist commonly omitted potentially important information about the
Table 3 Documentation of observations, investigations and procedures on presentation in emergency department $(n=89)$

\begin{tabular}{ll}
\hline $\begin{array}{l}\text { Procedure or investigation on presentation of } \\
\text { unexpected infant or child death }\end{array}$ & $\begin{array}{l}\text { Documented in records } \\
\mathbf{n}(\%)\end{array}$ \\
\hline Initial multiagency meeting & $37(42)$ \\
\hline Blood toxicology & $28(31)$ \\
Urine toxicology & $19(21)$ \\
\hline Blood culture & $43(48)$ \\
Blood biochemistry & $43(48)$ \\
Cerebrospinal fluid sample & $31(35)$ \\
Carboxyhaemoglobin & $11(12)$ \\
Skin biopsy for fibroblast culture & $8(9)$ \\
Presence of livido & $41(46)$ \\
Examination by paediatrician & $61(69)$ \\
Optic fundi examined & $19(21)$ \\
Skeletal survey and results & $79(89)$ \\
\hline
\end{tabular}

circumstances of death (eg, sleeping position, position of baby or bed covers when found and position of infant in relation to others in the bed for bed-sharing deaths), and many did not list what investigations had been done (table 3). Few paediatricians' letters or postmortem reports noted whether the results of ED investigations had been passed to the pathologist. Postmortem examinations were largely conducted by a paediatric pathologist alone or jointly with a forensic pathologist (table 4).

The postmortem protocol for sudden unexpected deaths of infants or children ${ }^{4}$ was usually followed quite closely, but there was poor documentation of the metabolic and genetic investigations. Details of documented postmortem investigations are shown in table 5 .

Neuropathology investigations were poorly documented; even in unexplained deaths of older children, there were only reports by neuropathologists for 22\% (5/23). Only 48\% (11/23) of reports mentioned histological examination of the hippocampus: eight of these were described as normal (including one by a neuropathologist) and three (two of which were examinations by a neuropathologist) were reported as showing the characteristic abnormality of the hippocampus.

There was no standard terminology used by pathologists in their reports to the coroners; recommendations from Kennedy et $a l^{4}$ or of chief coroner were not followed.

\section{Quality of care in reporting back to parents}

Only $15 \%(13 / 89)$ of the inquest reports mentioned a final multiagency review meeting, and it was documented in only 12 cases (where parents had been informed of the outcome of the meeting).

A final meeting with the paediatrician to explain the results of investigations was reported by $61 \%$ (54/89) of families; four families reported being offered such a meeting but had not taken it up.

Table 4 Pathologists involved in postmortem examinations

\begin{tabular}{ll}
\hline Type of pathologist(s) & $\begin{array}{l}\text { Cases } \\
\mathbf{n}(\%)\end{array}$ \\
\hline Paediatric and perinatal pathologist alone & $76(85)$ \\
\hline Forensic pathologist alone & $4(5)$ (all for children aged >1 year) \\
\hline Adult pathologist alone & $1(1)$ \\
\hline Paediatric pathologist+forensic pathologist & $8(9)$ \\
\hline
\end{tabular}


Table 5 Documentation of investigations and results at postmortem examination

\begin{tabular}{ll}
\hline Investigation & $\begin{array}{l}\text { Cases documented with } \\
\text { results } \\
\mathbf{n}(\%)\end{array}$ \\
\hline Toxicology & $51(57)$ \\
\hline Microbiology and virology & $82(92)$ \\
\hline Full histological investigations (to RCPath protocol) & $88(99)$ \\
\hline
\end{tabular}

The frequency of documentation of a multi-agency meeting $(15 \%$ vs $14 \%)$ or a final meeting with a paediatrician $(62 \%$ vs $61 \%$ ) did not change before and after the end of 2013, respectively.

\section{Classification of cause of death}

Most deaths included in this study went to inquest: $70 \%(42 / 60)$ of the SIDS deaths and $61 \%(14 / 23)$ of the SUDC deaths, though some coroners did not hold an inquest when the pathologist attributed death to a 'natural' cause such as 'SUDI' or 'SIDS'.

The nomenclature used for the final certified cause of death was dependent on the individual coroner rather than any consistent or systematic approach between coroners. Of the 10 deaths of infants under 1 year of age for which no significant contributory factors were identified in our multiprofessional review meetings (ie, those classified as Avon level IA or IB: see table 1), which thus unequivocally met the definition of SIDS, ${ }^{6}$ four were certified as SIDS, two as SUDI, two as 'natural causes' and two as 'unascertained' by the coroners. Four families reported that they had been informed that, as their baby had died in bed with an adult, the death could not be categorised as SIDS, and four pathology reports (including two of those mentioned by parents) also contained statements to this effect.

In $79 \%$ cases $(72 / 91)$, the cause of death attributed by the coroner was the same as that of the pathologist; in 12 cases, the pathologist's cause of death of 'unascertained' or 'SIDS' was changed by the coroner to 'natural causes'; in 3 cases, the pathologist's cause of death of 'SIDS' was changed by the coroner to 'unascertained'; in 1 case, the pathologist's cause of death of 'unascertained' was changed to 'SIDS' by the coroner, and in 2 cases specific infectious causes of death given by a forensic pathologist were changed to 'SUDC' by the coroner. In both cases, the study multiprofessional review panel agreed with the coroner that there was insufficient evidence to attribute the death to infection.

Initial home visit by health professional

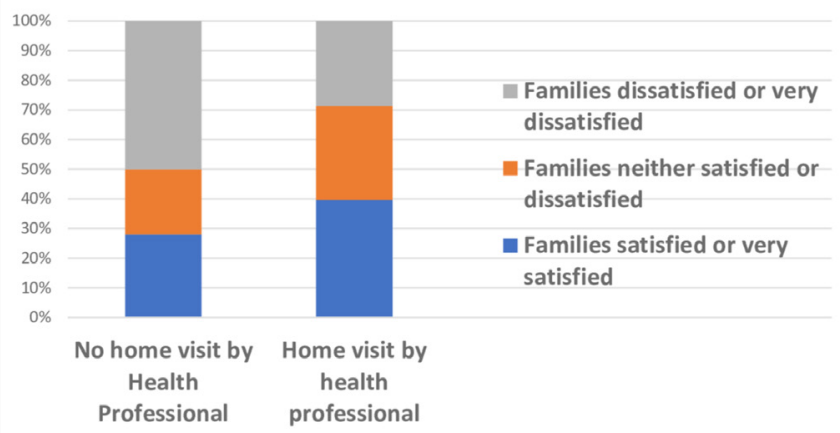

Figure 2 Level of satisfaction with the overall investigative procedures if they did or did not have an initial home visit by a health professional.
Final meeting with the Paediatrician.

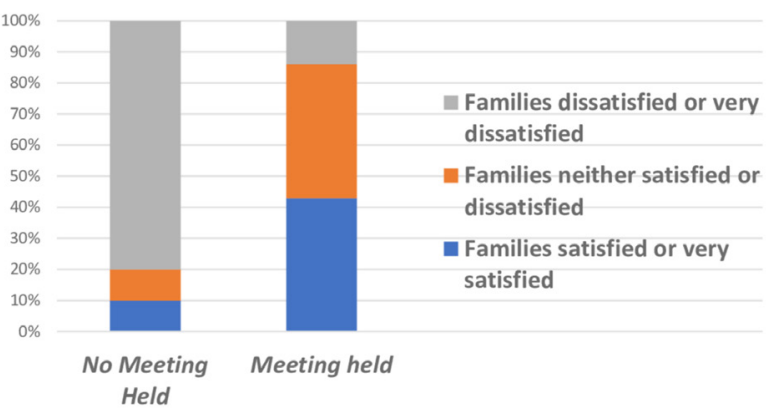

Figure 3 Level of satisfaction with the overall investigative procedure if families did or did not have a final meeting with the paediatrician.

\section{Families' satisfaction with the process}

Several families expressed concern at the way they had been treated by some staff (including clinical staff, police, social care staff or coroners' staff) though specific members of staff were also singled out by families as having provided exceptional care or support.

Figure 2 shows parental satisfaction with the investigative process and whether an initial home visit by a healthcare professional had taken place, and figure 3 shows parental satisfaction and whether a final meeting with the paediatrician was held. Families valued both the initial home visit and the final meeting very highly. Figure 4 shows that parents' level of satisfaction with the whole investigative process changed little between 2007-2013 and 2014-2017.

\section{DISCUSSION}

Since 2008, the Statutory Guidance to the Children Act has required that the full results of the local child death review process, including the final multiagency review meeting, be provided to the coroner for the conduct of the inquest after unexpected infant or child deaths. ${ }^{245}$ We found that the information provided to coroners was commonly lacking in some investigation results. Few inquests had the benefit of a full account of the multiagency discussions and conclusions from the final local case review meeting.

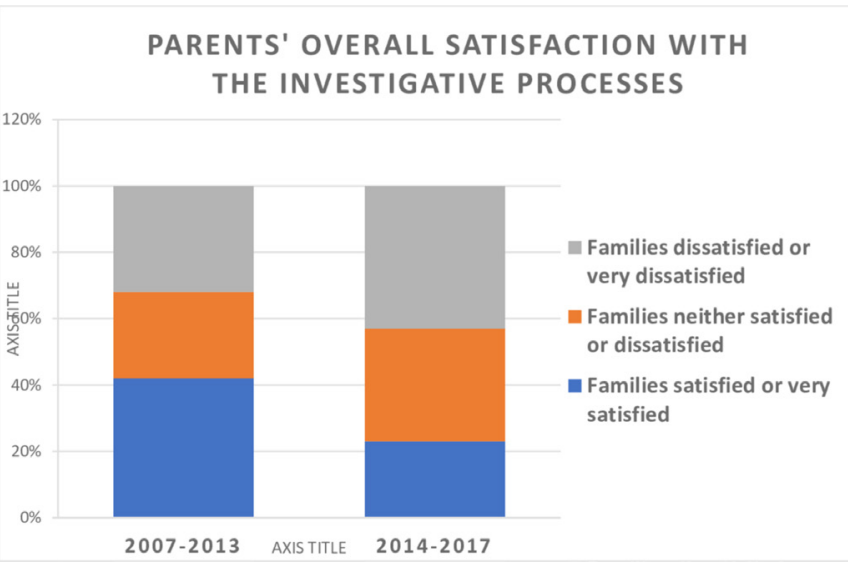

Figure 4 Level of satisfaction with the overall investigative procedure and changes over time. 
The categorisation of the 'cause' of death by pathologists and coroners among the unexplained infant and child deaths varied widely and was largely determined by the views of individual coroners and pathologists rather than established criteria. Similarly, Garstang et $\mathrm{al}^{9}$ in the West Midlands found that pathologists and coroners were more likely to label cases as 'undetermined' than 'SIDS' even when internationally agreed criteria for SIDS were met.

We acknowledge the potential for recall bias to influence parents' recollection of the precise circumstances of their child's death. This emphasises the importance of accurate detailed contemporaneous recording of the scene and circumstances of all unexpected child deaths.

Despite the lack of some potentially important information in the documentation provided to the inquests, the parental narrative account and information made important contributions to our multiagency review meetings. This enabled us to achieve a unanimous consensus view on the Avon category for the deaths for $89 / 91$ of the cases reviewed.

Inquest documentation commonly did not include either a parents' narrative account or certain important information (eg, position baby put down to sleep and details of bedsharing arrangements). While unlikely to have affected the conclusion on the cause of death, this information would potentially have been important in discussing the death with parents and in providing focused support for future pregnancies and with future children.

The lack of contemporaneous documentation of the death scene in many instances (and our subsequent reliance on parental recall) limits our ability to identify potentially hazardous sleep circumstances accurately. Confidently attributing death to asphyxia, even in hazardous sleeping circumstances, is seldom possible even with immediate expert scene review. ${ }^{38}$

We therefore suggest that all unexpected and unexplained deaths of infants or children should be categorised on the death certificate (in a slight modification of the approach suggested by the chief coroner ${ }^{4}$ ), as 'unexplained sudden death in infancy' or 'unexplained sudden death in childhood' with, whenever appropriate, a note that findings are compatible with the diagnosis of SIDS $^{46}$ or SUDC, ${ }^{7}$, respectively.

In addition, a secondary categorisation for all unexpected deaths of infants or children should be determined at the Child Death Review Meeting ${ }^{5}$ using the Avon clinicopathological classification. $^{24}$

This would allow the collection by both the ONS (collects death certification data) and the National Child Mortality Database (collects child death review process data) of consistent, accurate and meaningful data on unexpected and unexplained deaths in infancy and childhood and facilitate provision of improved information and subsequent support to bereaved families.

The Avon classification allows the identification of possible or probable contributory factors to the death in several domains: medical, social, physical, environmental, microbiological, radiological, metabolic and so on. This may help ensure that appropriate support and supervision is offered in future pregnancies, particularly when there has been concern about the presence of neglect or inappropriate parenting decisions as potentially contributory factors to the death.

Data collection on all unexpected infant and child deaths by local Child Death Overview Panels has facilitated the recognition of some modifiable contributing factors. However, there has been no systematic detailed national data collection or national systematic audit of the quality or completeness of the investigations.
The limitations to our assessment of the quality of these investigations included recruiting through a parent support charity, the Lullaby Trust, which meant that only bereaved families who contacted the Trust (around 50\% nationally) were sampled. Only a small sample of unexpected deaths over this 10-year period were recruited, making it difficult to gauge the generalisability of the findings. There are gaps in the documented evidence and although these were often filled by the parental interviews, the time between our interview and the death introduces potential recall bias.

The families included in this study were self-selected and overall were less deprived and better educated than most bereaved families in our previous studies, although our approach of using specialist health professionals to conduct these sensitive interviews gave us a rich source of information on which to base our findings.

\section{CONCLUSIONS}

Despite the limitations of this study, the wide variation in the quality of care and support provided to bereaved families that we have identified, the lack of good communication either between agencies or with families and the relative lack of appropriate care and support from a paediatrician are areas of serious concern requiring better monitoring and audit to improve the care given to families.

The great value attached by families to the initial home visit by a health professional and the final meeting with the paediatrician emphasise the importance of paediatricians fully engaging with this process for all unexpected child deaths.

There is a general need for further joint multiagency training on responding to unexpected deaths of children.

The implementation, from April 2019, of a National Child Mortality Database will allow more detailed investigation of factors contributing to child deaths and continuing monitoring and audit of the nature and quality of investigations and the care of families after unexpected deaths of infants and children.

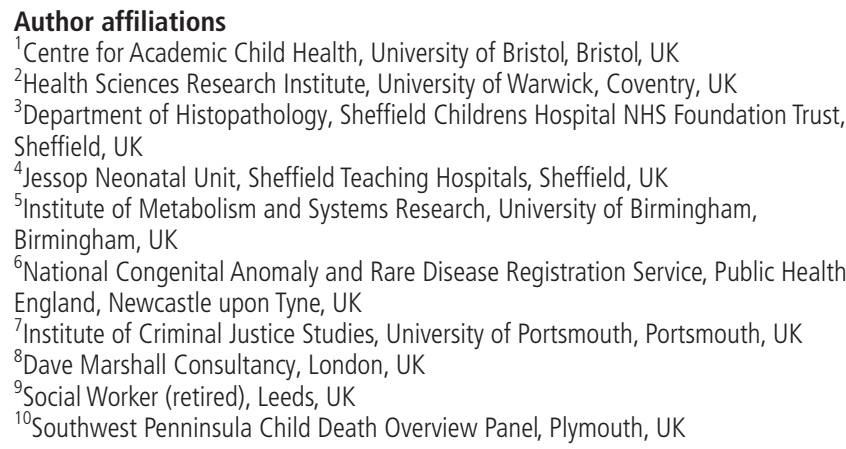

${ }^{6}$ National Congenital Anomaly and Rare Disease Registration Service, Public Health England, Newcastle upon Tyne, UK

${ }^{7}$ Institute of Criminal Justice Studies, University of Portsmouth, Portsmouth, UK ${ }^{8}$ Dave Marshall Consultancy, London, UK

${ }^{9}$ Social Worker (retired), Leeds, UK

${ }^{10}$ Southwest Penninsula Child Death Overview Panel, Plymouth, UK

Acknowledgements We are grateful to the bereaved families that consented to participate in this study, as well as the coroners and coroner officers who provided documents to us.

Contributors The study was designed by PF, PB, Jl, AP and AKE. The interviews with families were conducted by PF, PS, MCC, RCC and AKE. AP coordinated the data collected from families and coroners. Multidisciplinary reviews were carried out by PF, PS, MCC, RCC, AKE, MWP, JF, DM, AL and CE-C. Analysis was carried out by PB and PF. The article was drafted by PF with revisions from all co-authors. All coauthors gave their approval for the final version of the article to be submitted.

Funding This study was funded by a research grant (project 268) from the Lullaby Trust, with support from Teddy's Wish and other charitable funding agencies.

Competing interests None declared.

Patient consent for publication Not required.

Ethics approval This project was approved by the National Research Ethics Service Committee South West - Central Bristol (Reference 14/SW/1084. October 2014). 
Provenance and peer review Not commissioned; externally peer reviewed.

Data availability statement No data are available.

Open access This is an open access article distributed in accordance with the Creative Commons Attribution Non Commercial (CC BY-NC 4.0) license, which permits others to distribute, remix, adapt, build upon this work non-commercially, and license their derivative works on different terms, provided the original work is properly cited, appropriate credit is given, any changes made indicated, and the use is non-commercial. See: http://creativecommons.org/licenses/by-nc/4.0/.

ORCID iDs

Peter Fleming http://orcid.org/0000-0003-2521-5764

Jenny Ingram http://orcid.org/0000-0003-2366-008X

Marta C Cohen http://orcid.org/0000-0001-5534-444X

Andrew K Ewer http://orcid.org/0000-0002-3825-4781

Martin Ward Platt http://orcid.org/0000-0003-0536-5749

\section{REFERENCES}

1 Kennedy H, Epstein J, Fleming PJ, et al. Sudden unexpected death in infancy. A multiagency protocol for care and investigation. The Report of a working group convened by the Royal College of Pathologists and the Royal College of Paediatrics and Child Health. RCPath \& RCPCH, London, 2004. Available: www.rcpch.ac.uk

2 Fleming PJ, Blair PS, Sidebotham PD, et al. Investigating sudden unexpected deaths in infancy and childhood and caring for bereaved families: an integrated multiagency approach. BMJ 2004;328:331-4.
3 Working together to safeguard children, 2006. Available: https://webarchive. nationalarchives.gov.uk/20100408113130/http://www.dcsf.gov.uk/everychildmatters/ resources-and-practice//G00060/

4 Kennedy H. Bates H, Debelle G, Lishman S, et al. Sudden unexpected death in infancy and childhood. Multi-agency guidelines for care and investigation. The report of a working group convened by the Royal College of pathologists and endorsed by the Royal College of paediatrics and child health, 2016. Available: https://www.rcpath.org/uploads/assets/uploaded/af879a1b-1974-46929e002c20f09dc14c.pdf

5 Child Death Review, Statutory and operational Guidance. Department of health and social care, 2018. Available: https://www.gov.uk/government/publications/child-deathreview-statutory-and-operational-guidance-england

6 Willinger M, James LS, Catz C. Defining the sudden infant death syndrome (SIDS): deliberations of an expert panel Convened by the National Institute of child health and human development. Pediatric Pathology 1991;11:677-84.

7 Krous HF, Chadwick AE, Crandall L, et al. Sudden unexpected death in childhood: a report of 50 cases. Pediatr Dev Pathol 2005:8:307-19.

8 The Lullaby Trust. Sids and SUDC facts and figures. Available: https://www. lullabytrust.org.uk/wp-content/uploads/Facts-and-Figures-for-2015-released-2017. pdf

9 Garstang J, Ellis C, Griffiths F, et al. Unintentional asphyxia, SIDS, and medically explained deaths: a descriptive study of outcomes of child death review (CDR) investigations following sudden unexpected death in infancy. Forensic Sci Med Pathol 2016;12:407-15. 